\begin{tabular}{|c|c|}
\hline Title & Investigation of energy absorption by clustered gold nanoparticles \\
\hline Author(s) & $\begin{array}{l}\text { Kwon, Jihun; Sutherland, Kenneth; Makarova, A nastasia; Matsuura, Taeko; Hashimoto, Takay uki; Peng, Hao; Toshito, } \\
\text { Toshiyuki; U megaki, Kikuo; Shirato, Hiroki; Shimizu, Shinichi }\end{array}$ \\
\hline Citation & $\begin{array}{l}\text { Nuclear instruments \& methods in physics research section b-beam interactions with materials and atoms, 429, } 34.41 \\
\text { https://doi.org/10.1016/.nimb.2018.05.033 }\end{array}$ \\
\hline Issue Date & $2018-08-15$ \\
\hline Doc URL & http:/hdl.handle.net/2115//9061 \\
\hline Rights & $\begin{array}{l}\text { (1) 2018. Ins manuscript verssion is made avallable under the CC-BY-IC-IVD } 4.01 \text { IIcense } \\
\text { http://reativecommons.org/icenses/by-nc-nd/4.0/ }\end{array}$ \\
\hline Rights(URL) & http:/creativecommons.org/icenses/by-nc-nd/4.0/ \\
\hline Type & article (author version) \\
\hline File Information & Manuscript_Revise2_20180428_final.pdf \\
\hline
\end{tabular}

Instructions for use 


\section{Investigation of energy absorption by clustered gold nanoparticles}

Jihun Kwon, $\mathbf{M S c}{ }^{\mathrm{a}}$, Kenneth Sutherland, $\mathbf{P h D}^{\mathrm{b}}$, Anastasia Makarova, $\mathbf{P h D}^{\mathbf{b}}$,

Taeko Matsuura, $\mathbf{P h D}{ }^{\mathrm{b}, \mathrm{e}}$, Takayuki Hashimoto, MD, $\mathbf{P h D}{ }^{\mathrm{c}}$, Hao Peng, $\mathbf{P h D}{ }^{\mathrm{b}, \mathrm{f}}$,

Toshiyuki Toshito, $\mathbf{P h D}{ }^{d}$, Kikuo Umegaki, $\mathbf{P h D}{ }^{\mathrm{b}, \mathrm{e}}$, Hiroki Shirato, $\mathrm{MD}, \mathbf{P h D}{ }^{\mathrm{b}, \mathrm{c}}$, and

Shinichi Shimizu, MD, $\mathbf{P h D}^{\mathrm{b}, \mathrm{g}, *}$

aDepartment of Radiation Oncology, Graduate School of Medicine, Hokkaido University,

Sapporo, Japan

${ }^{\mathrm{b}}$ Global Station for Quantum Medical Science and Engineering, Global Institution for

Collaborative Research and Education (GI-CoRE), Hokkaido University, Sapporo, Japan

'Department of Radiation Medicine, Faculty of Medicine, Hokkaido University, Sapporo, Japan

${ }^{d}$ Department of Proton Therapy Physics, Nagoya City West Medical Center, Nagoya Proton

Therapy Center, Nagoya, Japan

eDivision of Quantum Science and Engineering, Faculty of Engineering, Hokkaido University

fDepartment of Radiation Oncology, Stanford University, Stanford, CA 94305, USA

gDepartment of Radiation Oncology, Faculty of Medicine, Hokkaido University, Sapporo, Japan 
*Corresponding Author: Shinichi Shimizu, M.D., Ph.D.

Department of Radiation Oncology, Hokkaido University Graduate School of Medicine

North 15, West 7, Kita-ku, Sapporo, Hokkaido, Japan.

Tel.: +81 (11) 7065977; fax: +81 (11) 7068638,

E-mail: sshing@med.hokudai.ac.jp 


\section{Abstract}

The utilization of gold nanoparticles (GNPs) as a radiation sensitizer has received broad attention.

Although GNPs form clusters in living cells, most previous simulation studies have assumed a homogeneous distribution of GNPs. In this study, a GNP cluster was constructed for simulations and the impact of cluster formation on dose enhancement was examined. Energy absorption by the GNPs was compared between clustered and homogeneous distributions for several different GNP concentrations and diameters under $100 \mathrm{keV}$ X-ray irradiations. Our simulations showed that clusters more efficiently absorbed the secondary electrons and photons produced by GNPs themselves. Furthermore, the impact of cluster formation on dose enhancement was more significant for smaller GNPs and higher concentrations. Our results suggest that previous simulations assuming a homogeneous GNP distribution have overestimated the dose enhancement, especially for smaller GNPs and higher concentrations. These findings should guide the selection of GNP size and concentration for effectively optimizing dose enhancement in future studies.

Keywords: gold nanoparticles; clustering; dose enhancement; radiation sensitizer; Monte Carlo simulation 


\section{Introduction}

The past twenty years have seen increasingly rapid advances in the field of nanotechnology in medicine. In particular, gold nanoparticles (GNPs) have especially attracted attention as a radiation sensitizer, principally due to three advantageous properties: easy surface functionalization; high biocompatibility; and high atomic number ( $\mathrm{Z}=79)$. GNPs are preferentially taken up in tumor cells when conjugated to peptides [1], antibodies [2] or polyethylene glycol (PEG) [3,4]. GNPs inside cells physically interact with therapeutic radiation and emit electrons, which cause local dose enhancement. These electrons or generated reactive oxygen species (ROS) damage critical structures such as DNA or mitochondria, leading to radiation sensitization [5-8]. Many groups have experimentally observed an increase of DNA double strand break yields in cells which contain GNPs under kV and MV X-ray irradiation [911]. Chithrani et al have investigated GNP size dependency on cell survival fraction [12]. An in vivo study performed by Hainfeld et al reported radiation sensitization and tumor regression with $1.9 \mathrm{~nm}$ diameter GNPs under $250 \mathrm{kVp}$ X-ray irradiation [13].

The dose enhancement induced by GNPs has been estimated using Monte Carlo simulations. 
Several studies have reported higher dose enhancement under lower energy X-ray irradiation since the dose enhancement is caused mainly by photoelectric and Auger electrons [14-17]. To date, several simulations have been carried out under the assumption that GNPs are homogeneously distributed inside the tumor cells [18-22]. However, previous in vitro experiments showed that GNPs actually form clusters [11,23-26]. When GNPs form clusters, the separation of GNPs becomes significantly smaller than that in homogeneous distributions and this closeness is expected to cause increased electron absorption by the surrounding ("bystander") GNPs. In other words, clustered GNPs would be expected to show lower dose enhancement than non-clustered GNPs. Recently, Zygmanski et al simulated the dose enhancement in simple planar array and slab cluster models and showed saturation of dose enhancement as the number of GNPs in the cluster increased [27]. Planar or hexagonally packed GNP clusters were previously investigated by other groups [28,29]. On the other hand, quantitative image analysis suggested that GNPs in clusters can actually become trapped inside vesicles [24]. By using parameters given by Peckys and De Jonge, Jeynes et al calculated the number of secondary electrons exiting the GNPs [30]. However, the direct cause of lower dose enhancement in clusters is the increased energy absorption by the surrounding GNPs instead of 
the surrounding water medium and the extent of this effect remains to be fully elucidated. We hypothesize that the energy absorption by the surrounding GNPs should be highly influenced by the inter-GNP separation in clusters.

Therefore, the objective of the present study is to evaluate the radiation absorption by clustered GNPs with use of a realistic model that enables us to investigate the effect of inter-GNP separation. We assess the impact of GNP size and concentration as determining factors of the GNP separation on energy absorption. Firstly, in a macroscopic study, we constructed a GNP cluster model using parameters reported previously [24] and compared the energy absorption between clustered and non-clustered GNPs. Then, we conducted a microscopic study with a simple two GNP model to better understand the energy absorption by the bystander GNPs as a function of GNP separation.

\section{Materials and Methods}

\subsection{General}

Macroscopic and microscopic studies were performed using, Geant4 (version 10.02.p02) Monte

Carlo simulation code [31,32]. Low energy electromagnetic physics list “Geant4-Penelope” was 
used to track low energy electrons. Energy cutoff lengths were $1 \mathrm{~nm}$ for all particles and the production threshold of secondary electrons was set to $250 \mathrm{eV}$. Atomic de-excitation processes, fluorescence emission, Auger electron emission, Auger cascade, and Particle Induced X-ray Emission (PIXE) were all activated. Monochromatic $100 \mathrm{keV}$ X-rays were chosen as the source radiation, because bombardment of polychromatic X-ray complicates analysis of the pure energy absorption phenomenon, especially in microscopic studies. The number of incident particles was $4.8 \times 10^{9}$ and $10^{8}$ for macroscopic and microscopic studies, respectively.

\subsection{Macroscopic study}

GNP distributions were simulated inside a cubic cell phantom filled with water, referring to the report by Peckys and De Jonge [24], in which quantitative measurements of cluster formation in living COS-7 cells were performed. Energy deposition to the whole volume of water medium and GNPs by physical interaction with X-rays is referred to as "energy absorption”, and was compared between clustered and non-clustered GNP distributions. Hereafter, these two types of spatial distributions are referred to as "Cluster" and "Homogeneous".

The volume of the COS-7 cell line used by Peckys and De Jonge was not reported [24]. 
Therefore, we derived the average volume from the BioNumbers website as $2016 \mu \mathrm{m}^{3}$ [33].

Each side of the cubic cell phantom was accordingly set to $12.6 \mu \mathrm{m}$. For Homogeneous simulations, spherical GNPs were randomly distributed within the cell phantom (Figure 1(a)). In contrast, for Cluster simulations, GNPs were placed inside a cluster sphere positioned at the center of the cell phantom (Figure 1(b)). The diameter of the cluster sphere was $4.6 \mu \mathrm{m}$. One hundred and sixty four spherical vesicles of $260 \mathrm{~nm}$ diameter were randomly distributed inside the cluster sphere and GNPs were randomly distributed within each vesicle (Figure 1(c)). Three diameters of GNPs (10, 30 and $50 \mathrm{~nm}$ ) were compared to evaluate GNP size dependency on energy absorption. The weight concentration of GNPs was set to $0.5,1,2$ or $3 \mathrm{mg} / \mathrm{mL}$ for both the Cluster and Homogeneous simulations, enforcing a consistent number of GNPs in both cases. Table 1 summarizes the number of GNPs in the cell phantom and vesicle for each case. This specification of cluster geometry allows us to investigate the separation between GNPs for each diameter and weight concentration.

Monochromatic $100 \mathrm{keV}$ X-rays were shot in parallel from one side of the cell phantom. Although this energy is relatively low compared to the energy usually used in clinical examinations, it is relevant because therapeutic X-rays scatter deep inside the body [34], and the 
energy is higher than the K-shell energy of gold (80.7 keV).

The influence of clustering was evaluated for GNPs with the same weight concentration and diameter by the Clustering Factor. Clustering Factor is given by the ratio of energy absorption by GNPs between Cluster and Homogeneous (non-clustered) distributions, as follows:

$$
\text { Clustering Factor }=\frac{\text { Energy absorbed by clustered GNPs }}{\text { Energy absorbed by non-clustered GNPS }}
$$

Thus, by definition, this factor will increase if additional energy absorption is measured in Cluster compared with Homogeneous distributions. Next, to evaluate the fraction of energy absorbed by the GNPs compared with the whole cell phantom, the macroscopic Relative Energy Absorption (REA Macro) was defined for both Cluster and Homogeneous GNP distributions. $\mathrm{REA}_{\text {Macro }}$ is given as the ratio of energy absorbed by GNPs to the total energy absorbed in the whole region of interest (i.e. all the water and GNPs inside the cubic cell phantom) as follows:

$$
\begin{aligned}
& R E A_{\text {Macro }}[\%] \\
& =\frac{\text { Energy absorbed by GNPs }}{\text { Energy absorbed by water medium }+ \text { Energy absorbed by GNPS }} \times 100
\end{aligned}
$$

Note: Clustering factor (Equation 1) does not consider absorption by water.

\subsection{Microscopic study}

The main aim of the microscopic study was to gain a better understanding of the mechanisms 
behind the macroscopic behavior described above, by analyzing the energy absorption by bystander GNPs in a simple two-GNP geometry. Hereafter, the closest GNP from the source GNP of interest is referred to as "Bystander GNP”. Cylindrical GNPs were used here instead of spherical GNPs to clarify how the energy absorption changes as a function of inter-GNP separation; and hence, the distance from source GNP surface to the bystander GNP. The use of cylinders enables us to fix the distance between the flat base of the source GNP cylinder and the base of the bystander GNP to a constant value (if spheres were used, surface curvature would enforce a variable separation between GNPs). The utilization of cylindrical (rod-shaped) GNPs as a radiation sensitizer has already been extensively studied for both in vitro and in vivo studies $[35,36]$.

A cylindrical GNP was first positioned at the center of the same cubic cell phantom used for the macroscopic simulations. $100 \mathrm{keV}$ X-rays were incident on the GNP from a disc source, connected to the surface of the GNP and the same diameter as the GNP. The energy distributions of the electrons and secondary photons exiting the GNP were derived. Subsequently, these energy distributions were used as the source radiation; $10^{8}$ particles were shot at another (bystander) GNP, positioned along the central axis of the cylinder, 0 to $500 \mathrm{~nm}$ away from the 
disk source located at the center of the cell phantom. At each separation, energy deposition to the surrounding water in the cell phantom and the energy absorption by the bystander GNP was

calculated. The diameter and height of the GNPs was always the same as the diameter of the source and set to 10,30 or $50 \mathrm{~nm}$.

The energy absorption by the bystander GNP was evaluated by the microscopic Relative Energy Absorption (REA Micro), defined similarly to the REA Macro (Equation 2).

\section{Results}

\subsection{Macroscopic study}

We constructed a GNP cluster model by using experimental parameters which were reported previously [24] and compared the energy absorption by GNPs between Homogeneous and Cluster distributions. Figure 2 shows a bar graph of the energy absorption by GNPs for Homogeneous and Cluster types with 0.5, 1, 2 and $3 \mathrm{mg} / \mathrm{mL}$ weight concentration. Each bar is split into two, according to the locations where the secondary and higher order electrons or photons that transferred energy to the GNPs were generated. The energy deposited by the electrons and photons produced in the water (blue) was approximately constant at each 
concentration value for all GNP sizes and both spatial distributions. As expected, the energy absorption by electrons and photons generated in water linearly increased with GNP concentration. In contrast, the energy deposition to GNPs by electrons and photons produced in GNPs (yellow) was highly dependent on GNP size and spatial distribution, in addition to concentration. Consequently, the energy absorption of the electrons and photons generated inside GNPs (yellow) strongly influenced the overall absorption tendency.

The energy absorption by GNPs from electrons and photons generated only inside the GNPs (yellow in Figure 2) is plotted as a function of GNP concentration in Figure 3. Interestingly, the energy absorption in Homogeneous distributions was directly proportional to the GNP concentration at each GNP size (Figure 3, (a)). On the other hand, the energy absorption by GNPs in Cluster distributions (Figure 3, (b)) showed markedly higher values and a non-linear response compared with Homogeneous distributions. We attribute this additional energy absorption in Cluster distributions compared with Homogeneous distributions as being caused by the (bystander) GNPs positioned nearby the (source) GNPs from which the secondary electrons were produced. The nonlinear behavior was more pronounced as the GNP size was reduced. 
The influence of GNP size on energy absorption in Cluster distributions was evaluated by the Clustering Factor, as defined in Equation (1). Figure 4 depicts the Clustering Factor for GNP concentrations between 0.5 and $3 \mathrm{mg} / \mathrm{mL}$. The Clustering Factor increased as the GNP diameter decreased and $10 \mathrm{~nm}$ diameter GNPs yielded a substantially higher value compared to those of 30 and $50 \mathrm{~nm}$ diameter. This observation was accentuated at increased concentrations; the Clustering Factor for $10 \mathrm{~nm}$ diameter GNPs in $3 \mathrm{mg} / \mathrm{mL}$ concentrations was 1.28 and 1.37 times higher than that of 30 and $50 \mathrm{~nm}$, respectively, while it was only 1.16 and 1.20 times higher in 1 $\mathrm{mg} / \mathrm{mL}$ concentrations.

The fraction of the energy absorbed by GNPs was assessed by the REA Macro parameter, as

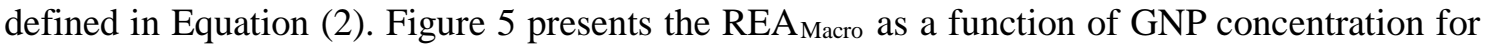

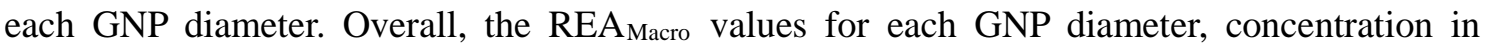
Cluster distributions were higher than those of Homogeneous distributions. However, we note that $\mathrm{REA}_{\text {Macro }}$ is calculated inside the cell phantom cube with $12.6 \mu \mathrm{m}$ sides. Although the

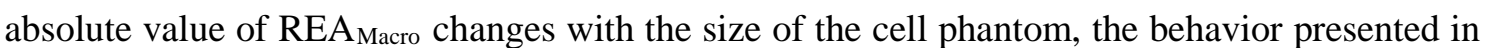
Figure 5 is not affected. 


\subsection{Microscopic study}

To gain a better understanding of the microscopic mechanisms underpinning the macroscopic behavior within our model, the energy absorption by bystander GNPs was analyzed in a simple microscopic geometry. Figure 6 shows $\mathrm{REA}_{\text {Micro }}$ as a function of the separation of the source and the bystander GNP surface. Larger GNPs showed higher REA Mirro values, but for all GNP

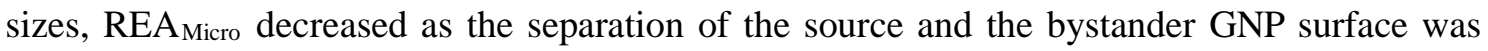
increased. The decline was initially steeper for smaller GNPs, likely because REA Micro depends significantly on the number of electrons incident on the bystander GNP, which is smaller for the smaller size of GNP at a given distance from the source.

Table 2 shows estimated separations of GNPs for Homogeneous and Cluster distributions, calculated by assuming that the GNPs and vesicles are cubic in shape. In all cases, the separation of GNPs in Homogeneous distributions was more than $140 \mathrm{~nm}$, but less than $50 \mathrm{~nm}$ in Cluster distributions. At constant weight concentration, the separation of GNPs reduced rapidly as the GNP diameter decreased because the number of GNPs increases with the cube of the side length. For example, the separation of GNPs in Homogeneous distributions with 3 $\mathrm{mg} / \mathrm{mL}$ concentration was 140,420 and $700 \mathrm{~nm}$ for 10,30 and $50 \mathrm{~nm}$ diameter GNPs, 
respectively. REA $A_{\text {Micro }}$ values for these separations were $0.08,0.14$ and less than $0.33 \%$ (700 nm

not calculated; $0.33 \%$ is the data at $500 \mathrm{~nm}$ separation). On the other hand, the separation of GNPs in Cluster distributions with $3 \mathrm{mg} / \mathrm{mL}$ concentration were 4, 11 and $18 \mathrm{~nm}$ for 10, 30 and

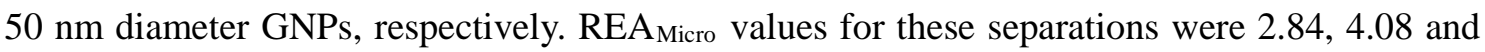
$5.73 \%$.

\section{Discussion}

We carried out both macroscopic and microscopic Monte Carlo simulations to reveal how GNP cluster formation affects the energy deposition to the GNPs and surrounding medium. Several groups previously investigated the effect on dose enhancement of clustered GNPs to the water medium, using models in which GNPs are densely packed and in direct contact with each other $[28,29]$. Our study modeled GNP clusters by using the parameters given by Peckys and De Jonge (who suggested that GNPs are packed inside vesicles) [24]. Jeynes et al previously calculated the number of secondary electrons exiting the GNPs by using those parameters given by Peckys and De Jonge [30]. In this study, we further calculated the energy deposition to surrounding GNPs and water. We have shown that the energy absorption by the GNPs was 
higher in Cluster compared with Homogeneous distributions (Figure 2). This is in agreement with the previous simulation studies indicating an overestimation of dose enhancement in homogeneously-distributed GNPs [27,37]. Similarly, McKinnon et al have reported the saturation of dose enhancement as a function of the thickness of a $\mathrm{Ta}_{2} \mathrm{O}_{5}$ nanoparticle shell around the cell nucleus [38].

Furthermore, we have discriminated the energy absorption by GNPs from secondary electrons and photons by the media in which they were generated. To the best of our knowledge, this discrimination approach was taken for the first time. We showed that the energy absorption from the secondary electrons and photons generated in the water was almost constant at the same concentration for all GNP sizes and spatial distributions (Figure 2). On the other hand, the energy absorption from electrons and photons generated in GNPs for Homogeneous and Cluster distributions was strongly related to GNP size and concentration. The observed linear increase of the energy absorption with GNP concentration in Homogeneous distributions (Figure 3, (a)) suggests that most of the energy absorption in Homogeneous distributions was brought about by self-absorption of electrons by GNPs. However, the non-linear and significantly higher energy absorption in Cluster distributions (Figure 3, (b)) predominantly originates from the energy 
absorption of secondary electrons and photons by bystander GNPs. These results indicate that the energy exchange among bystander GNPs in Cluster distributions is the main mechanism of the additional energy absorption compared to Homogeneous distributions. This energy exchange was neglected in several previous studies in which the dose enhancement was investigated by using heterogeneously distributed GNPs [39,40].

We also assessed the influence of GNP size on energy absorption. In previous studies, the dose enhancement was simulated with different-sized GNPs at fixed number of GNPs using a similar geometry to that used in the present paper [41]. On the other hand, we simulated the influence of GNP size at fixed weight concentrations because our hypothesis was that the inter-GNP separation should highly influence the energy absorption by clustered GNPs. Larger GNPs exhibited higher energy absorption in both the macroscopic and microscopic simulations (Figures 2, 3 and 6). This is because electrons have a higher tendency to escape smaller GNPs compared to larger GNPs. This trend is consistent with early findings in simulations $[27,40,42]$ and quantitative measurements using electron spin resonance (ESR) spectroscopy [43]. In contrast, smaller GNPs showed a higher Clustering Factor and this trend was enhanced for higher GNP concentrations (Figure 4), due to the increased energy absorption by bystander 
GNPs. Since the separation of GNPs become closer for smaller GNPs and higher concentrations

(Table 2), we can infer that the GNP separation significantly impacts the overall energy absorption by GNPs. This hypothesis was confirmed by our findings in the microscopic study that the REA $A_{\text {Micro }}$ was highly dependent on the separation of the source and the bystander GNPs (Figure 6). This microscopic observation is in agreement with a recent study which reported a decrease of the dose enhancement ratio (DER), defined as the ratio of the energy absorbed by the water medium in the presence and the absence of GNPs, with GNP separation [44]. This suggests that the influence of cluster formation on the dose enhancement is more significant in smaller GNPs at fixed weight concentration. As a clinical radiation sensitizer, smaller GNPs, such as $1.9 \mathrm{~nm}$ diameter, have been widely investigated owing to their high surface to volume ratio $[5,13,15,45,46]$. However, our findings indicate that dose calculations assuming a homogeneous distribution tend to overestimate the dose enhancement, especially in smaller GNPs.

In calculations of the dose enhancement, several groups have previously recorded the information of particles exiting GNPs in phase space files and utilized these data as a particle source $[40,47]$. Although this technique is useful to obtain radial dose distributions, it should be 
noted that a widely-accepted model of energy absorption by bystander GNPs has not been published to date. Furthermore, the dose enhancement has been reported to have angular anisotropy [48].

The target application of the present study is the accumulation of GNPs within the tumor cells through chemical functionalization, known as called "active targeting” [3,4]. Nevertheless, the results of this investigation are directly applicable to another method of GNP tumor targeting, "passive targeting”, which utilizes a tumor blood vessel's notable characteristic: the enhanced permeability and retention (EPR) effect [49]. In such a scenario, the concentration of GNPs inside the blood vessel is estimated to be about 20 times higher than that in the overall tumor volume [50]. We therefore hypothesize that energy absorption by bystander GNPs will be significant in the tumor blood vessels when GNPs are passively targeted.

Our study has several limitations that are the subject of ongoing investigation. First, we did not track the electrons after exiting the cell phantom because our focus was to calculate the influence of GNP clustering within a single cell. This geometrical limitation causes an underestimation of the energy absorbed by water, especially in Homogeneous distributions where some GNPs were positioned close to the border of the cell phantom. However, if this 


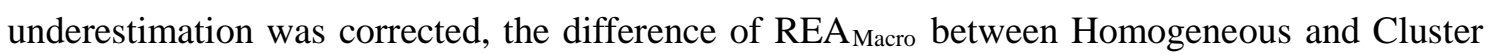
distributions would increase further since the energy absorption by the water contributes to the

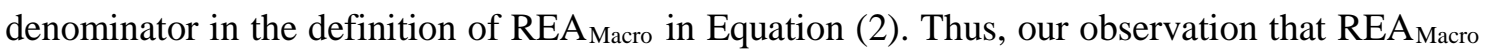
in Cluster was higher than that of Homogeneous distributions would be reinforced. For the same reason, calculating the DEF in the present geometry will not necessarily yield a result that reflects the real situation in tumors [41]. Second, GNP size dependency on cellular uptake was not considered. As such, the optimum GNP size in terms of uptake efficiency is still under investigation. Chithrani et al reported that $50 \mathrm{~nm}$ diameter GNPs show the highest internalization rate among 14, 50 and $74 \mathrm{~nm}$ diameter GNPs [25], while another group found that $20 \mathrm{~nm}$ diameter GNPs most efficiently leaked into tumors among 20, 40, 60, 80 and $100 \mathrm{~nm}$ GNPs [51]. In the present study, we assumed uniform uptake efficiency of GNPs to evaluate the energy absorption for constant weight densities. Third, we did not consider principal cellular structures such as the nucleus and mitochondria, which have been reported as potential targets of GNP-enhanced radiation therapy [45,52,53]. Future study should therefore focus on assessment of the energy deposition to specific structures based on precise biological measurements of GNP spatial distributions and increasingly realistic cell geometries. Finally, 
Geant4-DNA and discrete electron transport models for gold should be utilized in order to track low energy electrons [54-57].

\section{Conclusions}

In this Monte Carlo simulation study, we investigated how GNP cluster formation and size influences energy absorption by bystander GNPs. Our results indicate that clustered GNPs show higher energy absorption compared to homogeneously distributed GNPs because secondary electrons emitted by GNPs deposit their energy to nearby bystander GNPs in clusters. Additionally, the impact of cluster formation on the dose enhancement is most significant for smaller GNPs and higher concentrations due to the proximity of GNPs in the cluster. We conclude that previous simulation studies assuming homogeneous GNP distributions may have overestimated the dose enhancement, especially for small GNPs. As such, our findings should guide the selection of GNP size and concentration to effectively increase the dose enhancement in future studies.

\section{Acknowledgements}


This work was partially supported by Japanese Society for the Promotion of Science (JSPS)

KAKENHI Grant Number JP17J03616. The authors are deeply grateful to Dr. Tsukasa Aso and Dr. Neil J Stewart for their insightful comments and suggestions. 


\section{Figures}
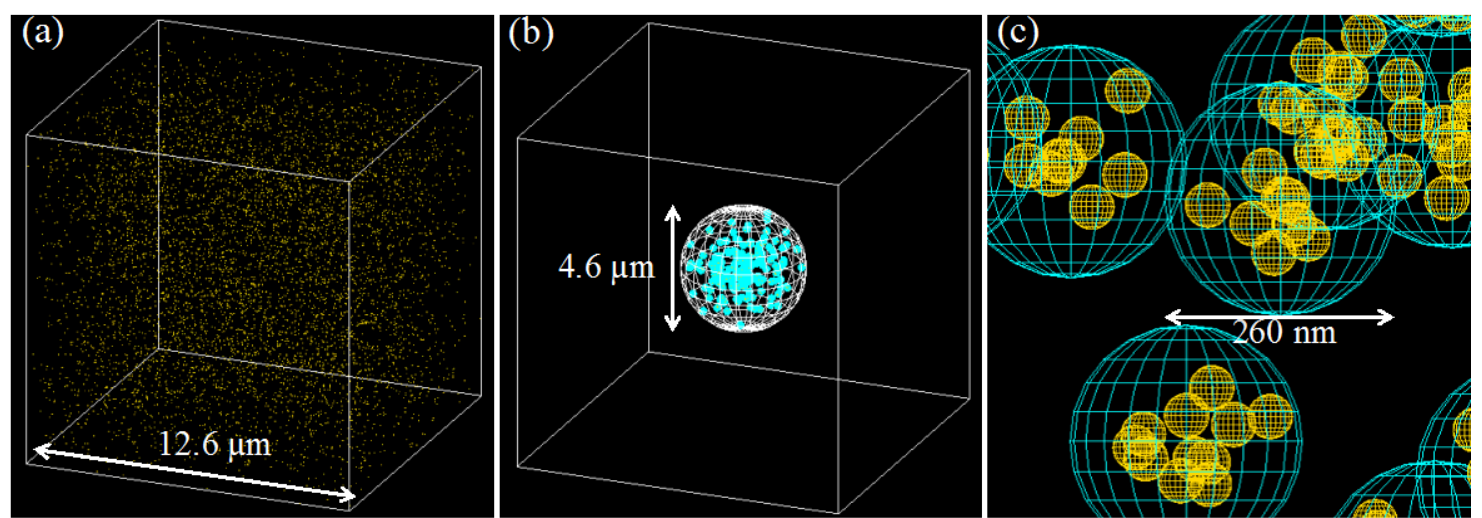

Figure. 1. Monte Carlo simulation geometry for the macroscopic study. (a): In the

Homogeneous simulation, spherical GNPs (yellow) were randomly distributed in a cubic cell

phantom, $12.6 \mu \mathrm{m}$ side length (white). (b): In the Cluster simulation, GNPs with 10, 30, or 50

nm diameter were randomly distributed within spherical vesicles (cyan, $260 \mathrm{~nm}$ diameter),

which in turn were randomly distributed within the spherical cluster sphere of $4.6 \mu \mathrm{m}$ diameter

(white sphere), which was positioned at the center of the same cell phantom as in (a). (c):

Enlarged view of GNPs distributed within cluster vesicles (260 nm diameter). The diameter of

GNPs shown is $50 \mathrm{~nm}$. 
(a) $0.5 \mathrm{mg} / \mathrm{mL}$

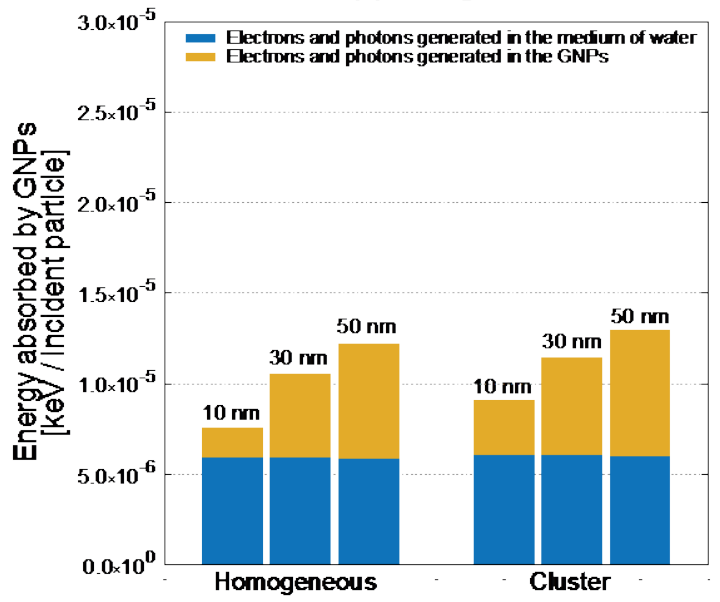

(c) $2 \mathrm{mg} / \mathrm{mL}$

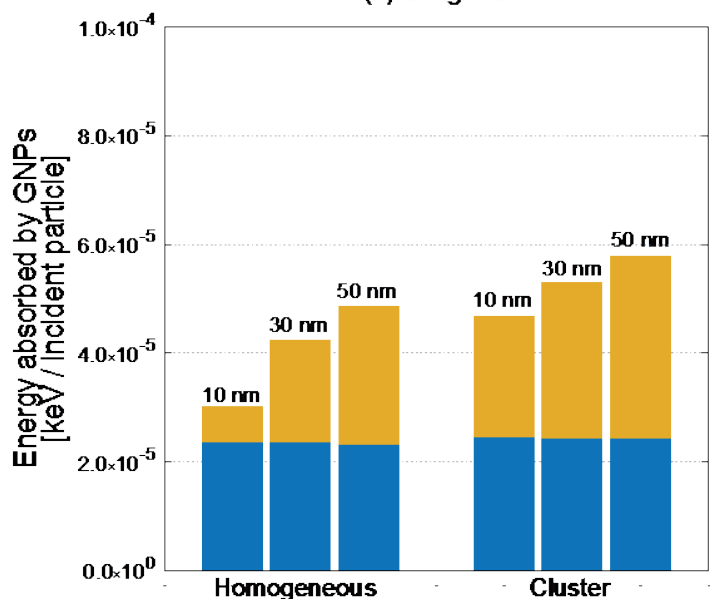

(b) $1 \mathrm{mg} / \mathrm{mL}$

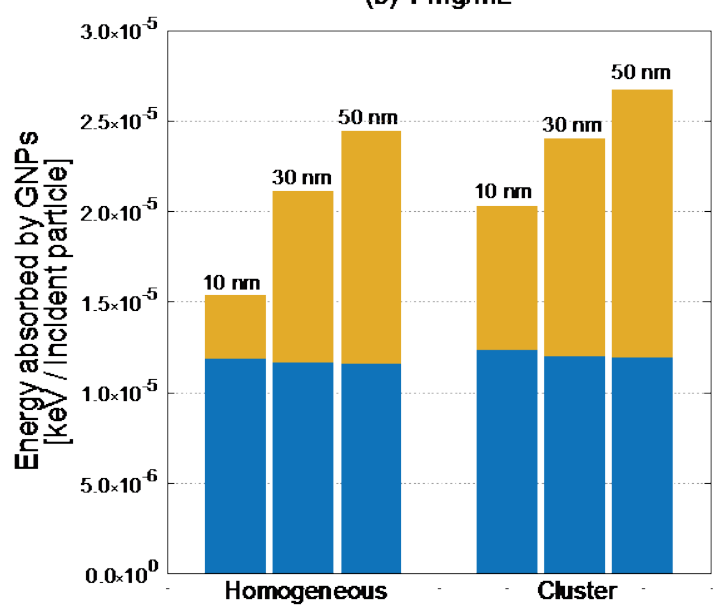

(d) $3 \mathrm{mg} / \mathrm{mL}$

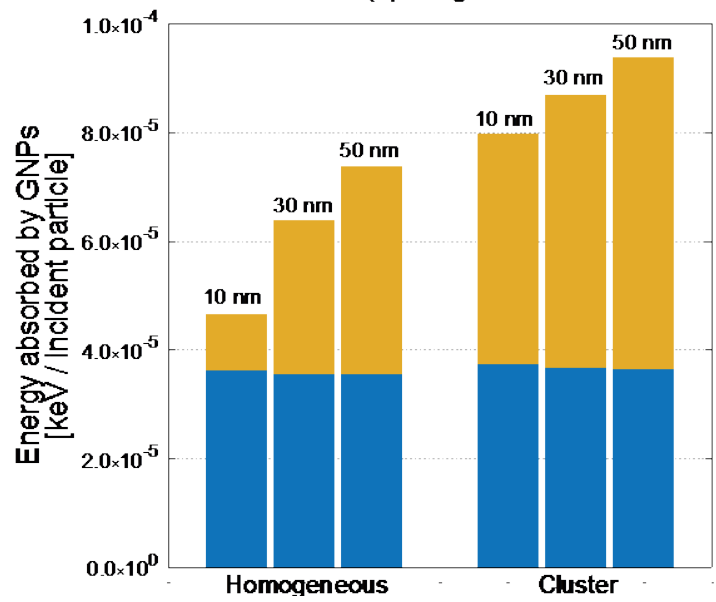

Figure. 2. Energy absorbed by GNPs in Homogeneous and Cluster distributions for 0.5 (a), 1

(b), 2 (c) and 3 (d) mg/mL weight concentrations. For all distributions and diameters of GNPs,

each bar is split into two according to the location (medium of water; blue or GNP; yellow)

where the secondary and higher order electrons or photons were generated. Energy absorbed by the GNPs from electrons and photons generated in GNPs (yellow) includes two types of energy absorption; (1) energy absorption by the same GNPs where the electrons and photons were generated (self-absorption), and (2) energy absorption by other GNPs which were positioned 
nearby the "source" GNPs where the electrons and photons were generated. 
(a) Homogeneous

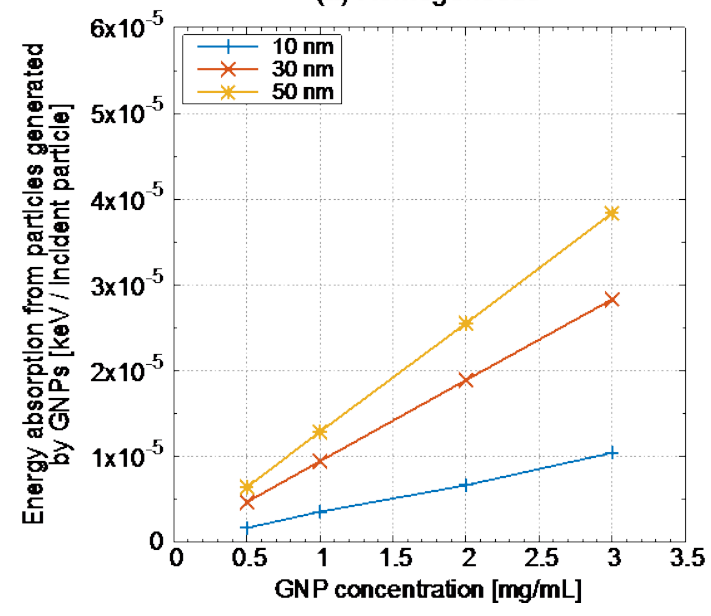

(b) Cluster

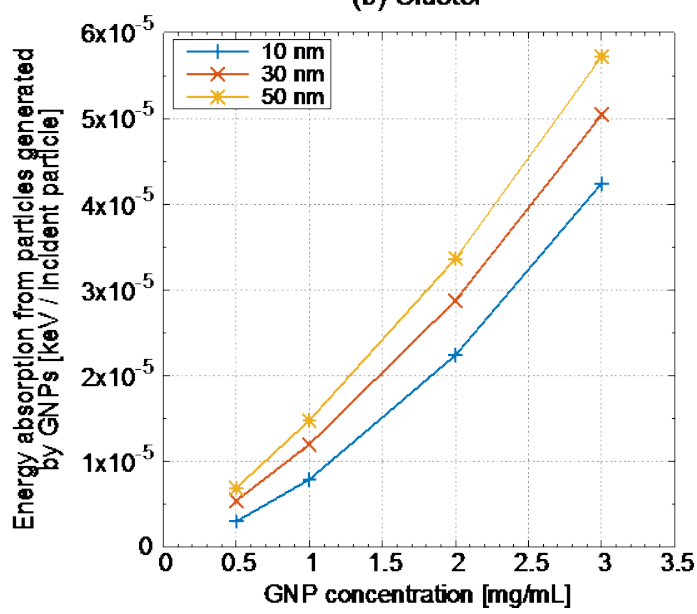

Figure. 3. GNP energy absorption from secondary and higher order electrons or photons generated by GNPs only (not water) for Homogeneous (a) and Cluster (b) distributions as a function of GNP concentration. Each value corresponds to the yellow bar in Figure 2 (energy absorption by GNPs from electrons and photons generated in the GNPs). 


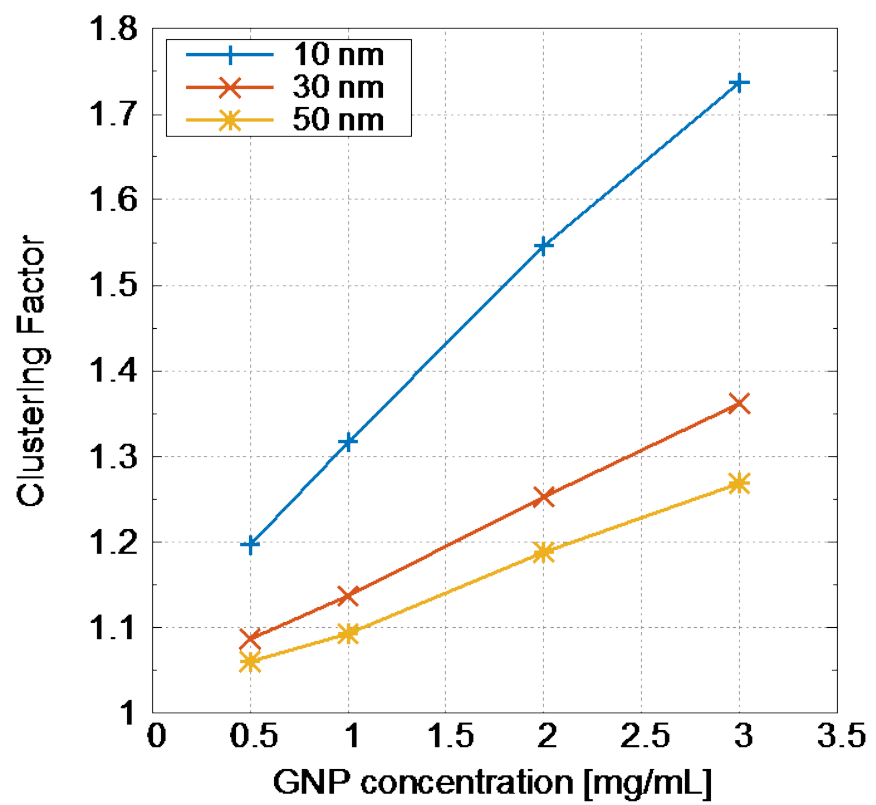

Figure. 4. Clustering Factor (defined by Equation 1) for each GNP diameter as a function of concentration. 
(a) Homogeneous

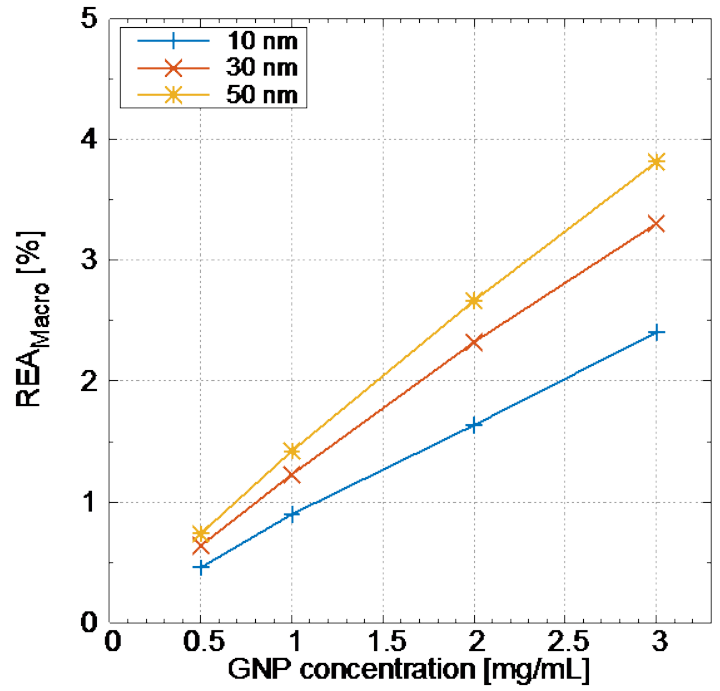

(b) Cluster

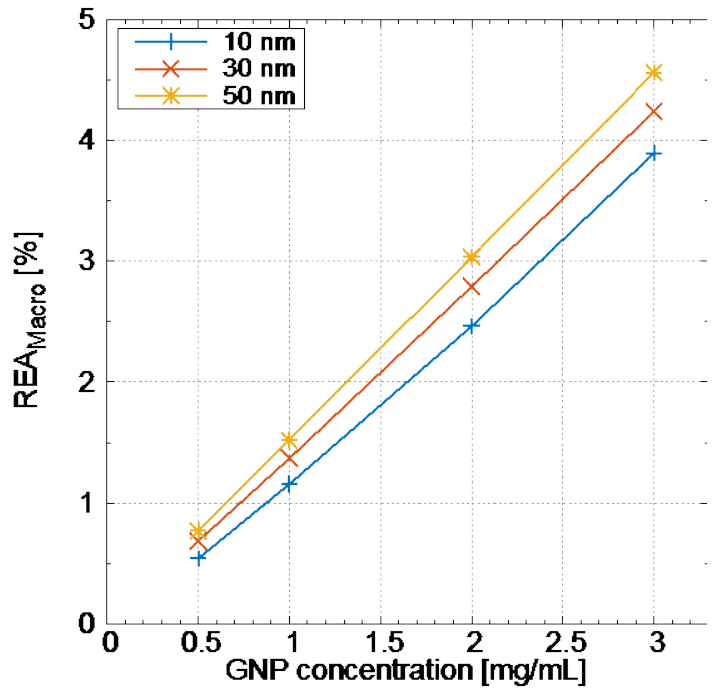

Figure. 5. REA $A_{\text {Macro }}$ (defined by Equation 2) for each GNP diameter for Homogeneous (a) and

Cluster (b) distributions, as a function of GNP concentration. 


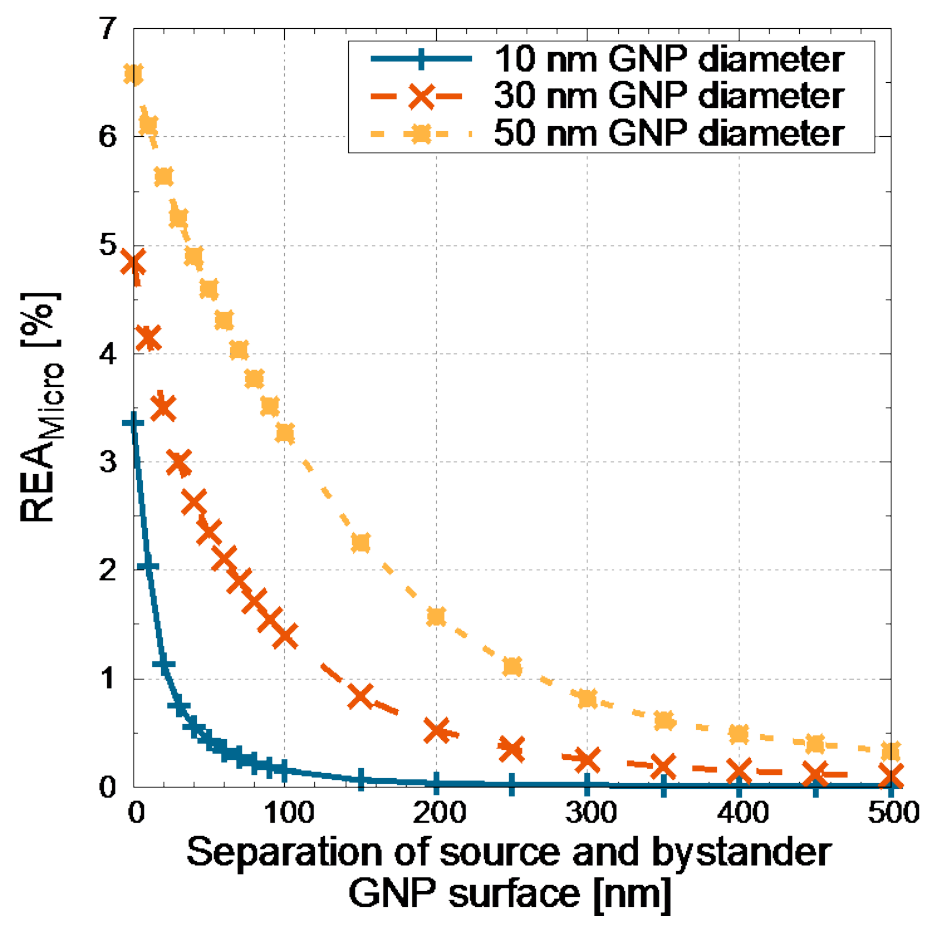

Figure. 6. The relationship between REA $A_{\text {Micro }}$ and the separation of source and the bystander

GNP surface. 


\section{Tables}

Table 1. Number of GNPs in the cell phantom and vesicle for each GNP diameter, concentration

\begin{tabular}{ccccccc}
\hline GNP diameter $[\mathrm{nm}]$ & \multicolumn{2}{c}{10} & \multicolumn{2}{c}{30} & \multicolumn{2}{c}{50} \\
\hline $\begin{array}{c}\text { Concentration } \\
{[\mathrm{mg} / \mathrm{mL}]}\end{array}$ & $\begin{array}{c}\text { Cell } \\
\text { phantom }\end{array}$ & Vesicle & $\begin{array}{c}\text { Cell } \\
\text { phantom }\end{array}$ & Vesicle & $\begin{array}{c}\text { Cell } \\
\text { phantom }\end{array}$ & Vesicle \\
0.5 & 99645 & 608 & 3691 & 23 & 797 & 5 \\
1 & 199290 & 1215 & 7381 & 45 & 1594 & 10 \\
2 & 398579 & 2430 & 14762 & 90 & 3189 & 19 \\
3 & 597869 & 3646 & 22143 & 135 & 4783 & 29 \\
\hline
\end{tabular}


Table 2. Estimated separation [nm] of cubic GNPs for each GNP weight concentration and GNP side length

\begin{tabular}{ccccccc}
\hline GNP side length $[\mathrm{nm}]$ & \multicolumn{2}{c}{10} & \multicolumn{2}{c}{30} & \multicolumn{2}{c}{50} \\
\hline $\begin{array}{c}\text { Concentration } \\
{[\mathrm{mg} / \mathrm{mL}]}\end{array}$ & $\begin{array}{c}\text { Homogen } \\
\text { eous }\end{array}$ & Cluster & $\begin{array}{c}\text { Homogen } \\
\text { eous }\end{array}$ & Cluster & $\begin{array}{c}\text { Homogen } \\
\text { eous }\end{array}$ & Cluster \\
0.5 & 263 & 15 & 788 & 44 & 1312 & 74 \\
1 & 206 & 10 & 619 & 29 & 1031 & 48 \\
2 & 162 & 6 & 485 & 17 & 700 & 28 \\
3 & 140 & 4 & 420 & 11 & 700 & 18 \\
\hline
\end{tabular}




\section{References}

[1] G. Wang, A.S. Norton, D. Pokharel, Y. Song, R.A. Hill, KDEL peptide gold nanoconstructs: Promising nanoplatforms for drug delivery, Nanomedicine Nanotechnology, Biol. Med. 9 (2013) 366-374. doi:10.1016/j.nano.2012.09.002.

[2] R.S. Riley, E.S. Day, Gold nanoparticle-mediated photothermal therapy : applications and opportunities for multimodal cancer treatment, (2017). doi:10.1002/wnan.1449.

[3] Arnida, M.M. Janát-Amsbury, A. Ray, C.M. Peterson, H. Ghandehari, Geometry and surface characteristics of gold nanoparticles influence their biodistribution and uptake by macrophages, Eur. J. Pharm. Biopharm. 77 (2011) 417-423.

doi:10.1016/j.ejpb.2010.11.010.

[4] I.C. Lin, M. Liang, T.Y. Liu, M.J. Monteiro, I. Toth, Cellular transport pathways of polymer coated gold nanoparticles, Nanomedicine Nanotechnology, Biol. Med. 8 (2012) 8-11. doi:10.1016/j.nano.2011.09.014.

[5] K.T. Butterworth, J. a Coulter, S. Jain, J. Forker, S.J. McMahon, G. Schettino, K.M. Prise, F.J. Currell, D.G. Hirst, Evaluation of cytotoxicity and radiation enhancement using 1.9 nm gold particles: potential application for cancer therapy., Nanotechnology. 21 (2010) 
295101. doi:10.1088/0957-4484/21/29/295101.

[6] M. Misawa, J. Takahashi, Generation of reactive oxygen species induced by gold nanoparticles under x-ray and UV Irradiations, Nanomedicine Nanotechnology, Biol. Med. 7 (2011) 604-614. doi:10.1016/j.nano.2011.01.014.

[7] F. Geng, K. Song, J.Z. Xing, C. Yuan, S. Yan, Q. Yang, J. Chen, B. Kong, Thio-glucose bound gold nanoparticles enhance radio-cytotoxic targeting of ovarian cancer., Nanotechnology. 22 (2011) 285101. doi:10.1088/0957-4484/22/28/285101.

[8] H.N. Tran, M. Karamitros, V.N. Ivanchenko, S. Guatelli, S. McKinnon, K. Murakami, T. Sasaki, S. Okada, M.C. Bordage, Z. Francis, Z. El Bitar, M.A. Bernal, J.I. Shin, S.B. Lee, P. Barberet, T.T. Tran, J.M.C. Brown, T.V. Nhan Hao, S. Incerti, Geant4 Monte Carlo simulation of absorbed dose and radiolysis yields enhancement from a gold nanoparticle under MeV proton irradiation, Nucl. Instruments Methods Phys. Res. Sect. B Beam Interact. with Mater. Atoms. 373 (2016) 126-139. doi:10.1016/j.nimb.2016.01.017.

[9] K.T. Butterworth, J.A. Wyer, M. Brennan-fournet, C.J. Latimer, M.B. Shah, F.J. Currell, D.G. Hirst, Variation of Strand Break Yield for Plasmid DNA Irradiated with High- Z Metal Nanoparticles, Radiat. Res. 387 (2008) 381-387. doi:10.1667/RR1320.1.Variation. 
[10] E. Brun, L. Sanche, C. Sicard-Roselli, Parameters governing gold nanoparticle X-ray radiosensitization of DNA in solution, Colloids Surfaces B Biointerfaces. 72 (2009) 128134. doi:10.1016/j.colsurfb.2009.03.025.

[11] D.B. Chithrani, S. Jelveh, F. Jalali, M. van Prooijen, C. Allen, R.G. Bristow, R.P. Hill, D. a. Jaffray, Gold Nanoparticles as Radiation Sensitizers in Cancer Therapy, Radiat. Res. 173 (2010) 719-728. doi:10.1667/RR1984.1.

[12] D.B. Chithrani, M. Dunne, J. Stewart, C. Allen, D.A. Jaffray, Cellular uptake and transport of gold nanoparticles incorporated in a liposomal carrier, Nanomedicine Nanotechnology, Biol. Med. 6 (2010) 161-169. doi:10.1016/j.nano.2009.04.009.

[13] J.F. Hainfeld, D.N. Slatkin, H.M. Smilowitz, The use of gold nanoparticles to enhance radiotherapy in mice, Phys. Med. Biol. 49 (2004) N309-N315. doi:10.1088/0031-9155/49/18/N03.

[14] B.L. Jones, S. Krishnan, S.H. Cho, Estimation of microscopic dose enhancement factor around gold nanoparticles by Monte Carlo calculations., Med. Phys. 37 (2010) 3809-3816. doi:10.1118/1.3455703.

[15] E. Lechtman, N. Chattopadhyay, Z. Cai, S. Mashouf, R. Reilly, J.P. Pignol, Implications 
on clinical scenario of gold nanoparticle radiosensitization in regards to photon energy, nanoparticle size, concentration and location., Phys. Med. Biol. 56 (2011) 4631-4647. doi:10.1088/0031-9155/57/1/291.

[16] M. Douglass, E. Bezak, S. Penfold, Monte Carlo investigation of the increased radiation deposition due to gold nanoparticles using kilovoltage and megavoltage photons in a 3D randomized cell model., Med. Phys. 40 (2013) 71710. doi:10.1118/1.4808150.

[17] S. Incerti, I. Istituto, F. Nucleare, M.A. Bernal, Simulation of Auger electron emission from nanometer-size gold targets using the Geant4 Monte Carlo simulation toolkit, Nucl. Instruments Methods Phys. Res. Sect. B. (2016). doi:10.1016/j.nimb.2016.02.005.

[18] S.H. Cho, Estimation of tumour dose enhancement due to gold nanoparticles during typical radiation treatments: a preliminary Monte Carlo study., Phys. Med. Biol. 50 (2005) N163-N173. doi:10.1118/1.1998660.

[19] S.H. Cho, B.L. Jones, S. Krishnan, The dosimetric feasibility of gold nanoparticle-aided radiation therapy (GNRT) via brachytherapy using low-energy gamma-/x-ray sources., Phys. Med. Biol. 54 (2009) 4889-4905. doi:10.1088/0031-9155/54/16/004.

[20] S.J. McMahon, W.B. Hyland, M.F. Muir, J. a. Coulter, S. Jain, K.T. Butterworth, G. 
Schettino, G.R. Dickson, A.R. Hounsell, J.M. O’Sullivan, K.M. Prise, D.G. Hirst, F.J.

Currell, Biological consequences of nanoscale energy deposition near irradiated heavy atom nanoparticles, Sci. Rep. 1 (2011) 1-10. doi:10.1038/srep00018.

[21] B. Koger, C. Kirkby, A method for converting dose-to-medium to dose-to-tissue in Monte Carlo studies of gold nanoparticle-enhanced radiotherapy, Phys. Med. Biol. 61 (2016) 2014-2024. doi:10.1088/0031-9155/61/5/2014.

[22] L. Strigari, V. Ferrero, G. Visonà, F. Dalmasso, A. Gobbato, P. Cerello, S. Visentin, A. Attili, Targeted dose enhancement in radiotherapy for breast cancer using gold nanoparticles, part 2: A treatment planning study, Med. Phys. 44 (2017) 1993-2001. doi:10.1002/mp.12178.

[23] W.N. Rahman, N. Bishara, T. Ackerly, C.F. He, P. Jackson, C. Wong, R. Davidson, M. Geso, Enhancement of radiation effects by gold nanoparticles for superficial radiation therapy, Nanomedicine Nanotechnology, Biol. Med. 5 (2009) 136-142. doi:10.1016/j.nano.2009.01.014.

[24] D.B. Peckys, N. De Jonge, Visualizing gold nanoparticle uptake in live cells with liquid scanning transmission electron microscopy, Nano Lett. 11 (2011) 1733-1738. 
doi:10.1021/nl200285r.

[25] B.D. Chithrani, A.A. Ghazani, W.C.W. Chan, Determining the size and shape dependence of gold nanoparticle uptake into mammalian cells, Nano Lett. 6 (2006) 662-668. doi:10.1021/nl052396o.

[26] S. Jain, J. a. Coulter, A.R. Hounsell, K.T. Butterworth, S.J. McMahon, W.B. Hyland, M.F. Muir, G.R. Dickson, K.M. Prise, F.J. Currell, J.M. O’Sullivan, D.G. Hirst, Cell-Specific Radiosensitization by gold nanoparticles at megavoltage radiation energies, Int. J. Radiat. Oncol. Biol. Phys. 79 (2011) 531-539. doi:10.1016/j.jjrobp.2010.08.044.

[27] P. Zygmanski, B. Liu, P. Tsiamas, F. Cifter, M. Petersheim, J. Hesser, E. Sajo, Dependence of Monte Carlo microdosimetric computations on the simulation geometry of gold nanoparticles., Phys. Med. Biol. 58 (2013) 7961-77. doi:10.1088/0031-9155/58/22/7961

[28] P. Zygmanski, W. Hoegele, P. Tsiamas, F. Cifter, W. Ngwa, R. Berbeco, M. Makrigiorgos, E. Sajo, A stochastic model of cell survival for high-Z nanoparticle radiotherapy., Med. Phys. 40 (2013) 24102. doi:10.1118/1.4773885.

[29] C. Kirkby, B. Koger, N. Suchowerska, D.R. McKenzie, Dosimetric consequences of gold 
nanoparticle clustering during photon irradiation, Med. Phys. (2017).

doi:10.1002/mp.12620.

[30] J.C.G. Jeynes, M.J. Merchant, a Spindler, a-C. Wera, K.J. Kirkby, Investigation of gold nanoparticle radiosensitization mechanisms using a free radical scavenger and protons of different energies, Phys. Med. Biol. 59 (2014) 6431-6443.

doi:10.1088/0031-9155/59/21/6431.

[31] S. Agostinelli, J. Allison, K. Amako, J. Apostolakis, H. Araujo, P. Arce, M. Asai, D. Axen, S. Banerjee, G. Barrand, F. Behner, L. Bellagamba, J. Boudreau, L. Broglia, a. Brunengo, H. Burkhardt, S. Chauvie, J. Chuma, R. Chytracek, G. Cooperman, G. Cosmo, P. Degtyarenko, a. Dell’Acqua, G. Depaola, D. Dietrich, R. Enami, a. Feliciello, C. Ferguson, H. Fesefeldt, G. Folger, F. Foppiano, a. Forti, S. Garelli, S. Giani, R.

Giannitrapani, D. Gibin, J.J. Gómez Cadenas, I. González, G. Gracia Abril, G. Greeniaus, W. Greiner, V. Grichine, a. Grossheim, S. Guatelli, P. Gumplinger, R. Hamatsu, K. Hashimoto, H. Hasui, a. Heikkinen, a. Howard, V. Ivanchenko, a. Johnson, F.W. Jones, J. Kallenbach, N. Kanaya, M. Kawabata, Y. Kawabata, M. Kawaguti, S. Kelner, P. Kent, a. Kimura, T. Kodama, R. Kokoulin, M. Kossov, H. Kurashige, E. Lamanna, T. 
Lampén, V. Lara, V. Lefebure, F. Lei, M. Liendl, W. Lockman, F. Longo, S. Magni, M.

Maire, E. Medernach, K. Minamimoto, P. Mora de Freitas, Y. Morita, K. Murakami, M.

Nagamatu, R. Nartallo, P. Nieminen, T. Nishimura, K. Ohtsubo, M. Okamura, S. O’Neale, Y. Oohata, K. Paech, J. Perl, a. Pfeiffer, M.G. Pia, F. Ranjard, a. Rybin, S. Sadilov, E. Di Salvo, G. Santin, T. Sasaki, N. Savvas, Y. Sawada, S. Scherer, S. Sei, V. Sirotenko, D. Smith, N. Starkov, H. Stoecker, J. Sulkimo, M. Takahata, S. Tanaka, E. Tcherniaev, E. Safai Tehrani, M. Tropeano, P. Truscott, H. Uno, L. Urban, P. Urban, M. Verderi, a. Walkden, W. Wander, H. Weber, J.P. Wellisch, T. Wenaus, D.C. Williams, D. Wright, T. Yamada, H. Yoshida, D. Zschiesche, Geant4-a simulation toolkit, Nucl. Instruments Methods Phys. Res. Sect. A Accel. Spectrometers, Detect. Assoc. Equip. 506 (2003) 250303. doi:10.1016/S0168-9002(03)01368-8.

[32] J. Allison, K. Amako, J. Apostolakis, H. Araujo, P.A. Dubois, M. Asai, G. Barrand, R.

Capra, S. Chauvie, R. Chytracek, G.A.P. Cirrone, G. Cooperman, G. Cosmo, G. Cuttone, G.G. Daquino, M. Donszelmann, M. Dressel, G. Folger, F. Foppiano, J. Generowicz, V.

Grichine, S. Guatelli, P. Gumplinger, A. Heikkinen, I. Hrivnacova, A. Howard, S. Incerti, V. Ivanchenko, T. Johnson, F. Jones, T. Koi, R. Kokoulin, M. Kossov, H. Kurashige, V. 
Lara, S. Larsson, F. Lei, F. Longo, M. Maire, A. Mantero, B. Mascialino, I. McLaren, P.M.

Lorenzo, K. Minamimoto, K. Murakami, P. Nieminen, L. Pandola, S. Parlati, L. Peralta, J.

Perl, A. Pfeiffer, M.G. Pia, A. Ribon, P. Rodrigues, G. Russo, S. Sadilov, G. Santin, T.

Sasaki, D. Smith, N. Starkov, S. Tanaka, E. Tcherniaev, B. Tomé, A. Trindade, P. Truscott,

L. Urban, M. Verderi, A. Walkden, J.P. Wellisch, D.C. Williams, D. Wright, H. Yoshida,

M. Peirgentili, Geant4 developments and applications, IEEE Trans. Nucl. Sci. 53 (2006)

270-278. doi:10.1109/TNS.2006.869826.

[33] R. Milo, P. Jorgensen, U. Moran, G. Weber, M. Springer, BioNumbers The database of key numbers in molecular and cell biology - BNID 101666, Nucleic Acids Res. 38 (2010) 750-753. doi:10.1093/nar/gkp889.

[34] A. Detappe, S. Kunjachan, P. Drané, S. Kotb, M. Myronakis, D.E. Biancur, T. Ireland, M.

Wagar, F. Lux, O. Tillement, R. Berbeco, Key clinical beam parameters for nanoparticle-mediated radiation dose amplification, Sci. Rep. 6 (2016) 34040.

doi:10.1038/srep34040.

[35] T. Wolfe, D. Chatterjee, J. Lee, J.D. Grant, S. Bhattarai, R. Tailor, G. Goodrich, P.

Nicolucci, S. Krishnan, Targeted gold nanoparticles enhance sensitization of prostate 
tumors to megavoltage radiation therapy in vivo, Nanomedicine Nanotechnology, Biol.

Med. 11 (2015) 1277-1283. doi:10.1016/j.nano.2014.12.016.

[36] T.D. Rane, A.M. Armani, Two-Photon Microscopy Analysis of Gold Nanoparticle Uptake in 3D Cell Spheroids, PLoS One. 11 (2016) e0167548.

doi:10.1371/journal.pone.0167548.

[37] M.P. Martinov, R.M. Thomson, Heterogeneous multiscale Monte Carlo simulations for gold nanoparticle radiosensitization, Med. Phys. 44 (2017) 644-653.

doi:10.1002/mp.12061.

[38] S. McKinnon, E. Engels, M. Tehei, K. Konstantinov, S. Corde, S. Oktaria, S. Incerti, M.

Lerch, A. Rosenfeld, S. Guatelli, Study of the effect of ceramic Ta2O5 nanoparticle

distribution on cellular dose enhancement in a kilovoltage photon field, Phys. Medica. 32

(2016) 1216-1224. doi:10.1016/j.ejmp.2016.09.006.

[39] C. Kirkby, E. Ghasroddashti, Targeting mitochondria in cancer cells using gold nanoparticle-enhanced radiotherapy: A Monte Carlo study, Med. Phys. 42 (2015) 1119. doi:10.1118/1.4906192.

[40] Y. Lin, S.J. McMahon, H. Paganetti, J. Schuemann, Biological modeling of gold 
nanoparticle enhanced radiotherapy for proton therapy., Phys. Med. Biol. 60 (2015) 414968. doi:10.1088/0031-9155/60/10/4149.

[41] P. Retif, A. Reinhard, H. Paquot, V. Jouan-Hureaux, A. Chateau, L. Sancey, M. Barberi-Heyob, S. Pinel, T. Bastogne, Monte Carlo simulations guided by imaging to predict the in vitro ranking of radiosensitizing nanoparticles, Int. J. Nanomedicine. 11 (2016) 6169-6179. doi:10.2147/IJN.S111320.

[42] M.K.K. Leung, J.C.L. Chow, B.D. Chithrani, M.J.G. Lee, B. Oms, D. a Jaffray, Irradiation of gold nanoparticles by x-rays: Monte Carlo simulation of dose enhancements and the spatial properties of the secondary electrons production., Med. Phys. 38 (2011) 624-631. doi:10.1118/1.3539623.

[43] E.J. Guidelli, O. Baffa, Influence of photon beam energy on the dose enhancement factor caused by gold and silver nanoparticles: An experimental approach., Med. Phys. 41 (2014) 32101. doi:10.1118/1.4865809.

[44] S.H. Ahn, K. Chung, J.W. Shin, W. Cheon, Y. Han, H.C. Park, D.H. Choi, Study on dependence of dose enhancement on cluster morphology of gold nanoparticles in radiation therapy using a body-centred cubic model, Phys. Med. Biol. 62 (2017) 7729-7740. 
doi:10.1088/1361-6560/aa87fd.

[45] L.E. Taggart, S.J. McMahon, K.T. Butterworth, F.J. Currell, G. Schettino, K.M. Prise, Protein disulphide isomerase as a target for nanoparticle-mediated sensitisation of cancer cells to radiation, Nanotechnology. 27 (2016) 215101. doi:10.1088/0957-4484/27/21/215101.

[46] E. Lechtman, J.-P. Pignol, Interplay between the gold nanoparticle sub-cellular localization, size, and the photon energy for radiosensitization, Sci. Rep. 7 (2017) 13268. doi:10.1038/s41598-017-13736-y.

[47] W. Sung, S.-J. Ye, A.L. McNamara, S.J. McMahon, J.F. Hainfeld, J. Shin, H.M. Smilowitz, H. Paganetti, J. Schuemann, Dependence of Gold Nanoparticle Radiosensitization on Cell Geometry, Nanoscale. (2017). doi:10.1039/C7NR01024A.

[48] S.M. Gadoue, D. Toomeh, P. Zygmanski, E. Sajo, Angular dose anisotropy around gold nanoparticles exposed to X-rays, Nanomedicine Nanotechnology, Biol. Med. 13 (2017) 1653-1661. doi:10.1016/j.nano.2017.02.017.

[49] Y. Matsumura, H. Maeda, A new concept for macromolecular therapeutics in cnacer chemotherapy: mechanism of tumoritropic accumulatio of proteins and the antitumor 
agents Smancs, Cancer Res. 46 (1986) 6387-6392. doi:10.1021/bc100070g.

[50] R.I. Berbeco, W. Ngwa, G.M. Makrigiorgos, Localized dose enhancement to tumor blood vessel endothelial cells via megavoltage X-rays and targeted gold nanoparticles: New potential for external beam radiotherapy, Int. J. Radiat. Oncol. Biol. Phys. 81 (2011) 270276. doi:10.1016/j.ijrobp.2010.10.022.

[51] S.D. Perrault, C. Walkey, T. Jennings, H.C. Fischer, W.C.W. Chan, Mediating tumor targeting efficiency of nanoparticles through design, Nano Lett. 9 (2009) 1909-1915. doi:10.1021/nl900031y.

[52] A.L. McNamara, W.W.Y. Kam, N. Scales, S.J. McMahon, J.W. Bennett, H.L. Byrne, J. Schuemann, H. Paganetti, R. Banati, Z. Kuncic, Dose enhancement effects to the nucleus and mitochondria from gold nanoparticles in the cytosol, Phys. Med. Biol. 61 (2016) 5993-6010. doi:10.1088/0031-9155/61/16/5993.

[53] M. Ghita, S.J. McMahon, L.E. Taggart, K.T. Butterworth, G. Schettino, K.M. Prise, A mechanistic study of gold nanoparticle radiosensitisation using targeted microbeam irradiation, Sci. Rep. 7 (2017) 44752. doi:10.1038/srep44752.

[54] Y. Lin, S.J. McMahon, M. Scarpelli, H. Paganetti, J. Schuemann, Comparing gold 
nano-particle enhanced radiotherapy with protons, megavoltage photons and kilovoltage photons: a Monte Carlo simulation, Phys. Med. Biol. 59 (2014) 7675-7689. doi:10.1088/0031-9155/59/24/7675.

[55] W. Sung, S. Jung, S.-J. Ye, Evaluation of the microscopic dose enhancement for nanoparticle-enhanced Auger therapy, Phys. Med. Biol. 61 (2016) 7522-7535. doi:10.1088/0031-9155/61/21/7522.

[56] D. Sakata, S. Incerti, M.C. Bordage, N. Lampe, S. Okada, D. Emfietzoglou, I. Kyriakou, K. Murakami, T. Sasaki, H. Tran, S. Guatelli, V.N. Ivantchenko, An implementation of discrete electron transport models for gold in the Geant4 simulation toolkit, J. Appl. Phys. 120 (2016) 244901. doi:10.1063/1.4972191.

[57] J. Allison, K. Amako, J. Apostolakis, P. Arce, M. Asai, T. Aso, E. Bagli, A. Bagulya, S. Banerjee, G. Barrand, B.R. Beck, A.G. Bogdanov, D. Brandt, J.M.C. Brown, H. Burkhardt, P. Canal, D. Cano-Ott, S. Chauvie, K. Cho, G.A.P. Cirrone, G. Cooperman,

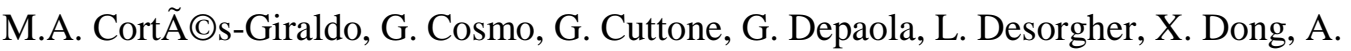
Dotti, V.D. Elvira, G. Folger, Z. Francis, A. Galoyan, L. Garnier, M. Gayer, K.L. Genser,

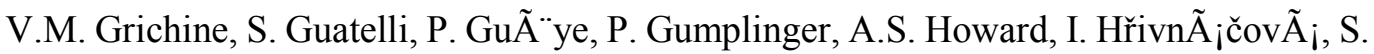


Hwang, S. Incerti, A. Ivanchenko, V.N. Ivanchenko, F.W. Jones, S.Y. Jun, P. Kaitaniemi,

N. Karakatsanis, M. Karamitrosi, M. Kelsey, A. Kimura, T. Koi, H. Kurashige, A.

Lechner, S.B. Lee, F. Longo, M. Maire, D. Mancusi, A. Mantero, E. Mendoza, B. Morgan,

K. Murakami, T. Nikitina, L. Pandola, P. Paprocki, J. Perl, I. Petrović, M.G. Pia, W.

Pokorski, J.M. Quesada, M. Raine, M.A. Reis, A. Ribon, A. Ristić Fira, F. Romano, G.

Russo, G. Santin, T. Sasaki, D. Sawkey, J.I. Shin, I.I. Strakovsky, A. Taborda, S. Tanaka,

B. TomÃ@, T. Toshito, H.N. Tran, P.R. Truscott, L. Urban, V. Uzhinsky, J.M. Verbeke,

M. Verderi, B.L. Wendt, H. Wenzel, D.H. Wright, D.M. Wright, T. Yamashita, J. Yarba,

H. Yoshida, Recent developments in GEANT4, Nucl. Instruments Methods Phys. Res.

Sect. A Accel. Spectrometers, Detect. Assoc. Equip. 835 (2016) 186-225.

doi:10.1016/j.nima.2016.06.125. 